\title{
Cerebellar and Hippocampal Activation during Eyeblink Conditioning Depends on the Experimental Paradigm: A MEG Study
}

\author{
Peter Kirsch, ${ }^{1,2}$ Caroline Achenbach,,${ }^{1,3}$ Martina Kirsch, ${ }^{3}$ Matthias Heinzmann, ${ }^{4}$ \\ Anne Schienle ${ }^{1,2}$ and Dieter Vaitl ${ }^{1,2}$ \\ ${ }^{1 \pm}$ Department of Clinical \& Physiological Psychology and ${ }^{2}$ Bender Institute of Neuroimaging, \\ University of Giessen, Germany; ${ }^{3}$ Departments of Neurology and ${ }^{4}$ Psychology \\ University of Heidelberg, Germany
}

\section{SUMMARY}

The cerebellum and the hippocampus are key structures for the acquisition of conditioned eyeblink responses. Whereas the cerebellum seems to be crucial for all types of eyeblink conditioning, the hippocampus appears to be involved only in complex types of learning. We conducted a differential conditioning study to explore the suitability of the design for magnetencephalography (MEG). In addition, we compared cerebellar and hippocampal activation during differential delay and trace conditioning. Comparable conditioning effects were seen in both conditions, but a greater resistance to extinction for trace conditioning. Brain activation differed between paradigms: delay conditioning provoked activation only in the cerebellum and trace conditioning only in the hippocampus. The results reflect differential brain activation patterns during the two types of eyeblink conditioning.

This research was conducted at the Department of Clinical \& Physiological Psychology, Otto-Behaghel Str. 10, D-35394 Giessen, Germany. Reprint requests to: Dr. Peter Kirsch e-mail: peter.kirsch@psychol.uni-giessen.de

\section{KEYWORDS}

delay conditioning, trace conditioning, brain electric source analysis

\section{INTRODUCTION}

Classical conditioning of eyeblink responses is one of the most frequently investigated paradigms. The experimental design consists of a tone or light as the conditioned stimulus (CS) and a corneal airpuff as the unconditioned stimulus (US). Since the occurrence of the conditioned response (CR) is very stable over time, the paradigm seems to be suitable for the investigation of magnetencephalography (MEG) correlates of human learning, where, due to an adverse signal to noise ratio, many trials are required to average an evoked response.

Animal studies of eyeblink conditioning have been quite consistent in demonstrating the importance of the cerebellum and the hippocampus for the acquisition and initiation of the CR (see Thompson \& Krupa, 1994; Woodruff-Pak \& Steinmetz, 2000 for an overview). Similar findings have been obtained for humans. In studies on patients with cerebellar lesions, it was shown that they are not able to generate conditioned eyeblink responses (Gerwig et al., 2003; Bracha et al., 1997; Daum et al., 1993). Brain imaging studies 
also point to the role the cerebellum plays in human eyeblink conditioning. Cerebellar activation has been found with both, position emission tomography (PET, Logan \& Grafton, 1995; Blaxton et al, 1996; Schreurs et al., 1997) and functional magnetic resonance imaging (fMRI, Ramnani et al., 2000). PET studies, however, have a number of methodological disadvantages making the generalization of the results difficult. The conditioning trials have to be presented in a block design, and because of the poor temporal resolution, no conclusion can be drawn about the time course of the cerebellar activation during learning. Even in fMRI studies with a better temporal resolution, in which event-related designs allow a single trial presentation, a temporal differentiation of the activation is hardly possible. Therefore, a method like the whole-head MEG, which provides both an excellent temporal and a sufficient spatial resolution, might allow additional insight into the neural basis of human eyeblink conditioning.

In contrast to the cerebellum, the role of the hippocampus in eyeblink conditioning is less clear. Although some studies found hippocampus unit responses in $\mathrm{CA} 1$ as well as in CA3 neurons (Berger \& Thompson, 1978; Berger et al., 1983) during delay conditioning, most researchers agree that delay conditioning is hippocampus independent (Steinmetz, 2000; Thompson \& Krupa, 1994). In contrast, for trace conditioning, and intact hippocampus is essential. Weiss et al. (1999), as well as many others, found an impairment of conditioned responses in rats after a hippocampal lesion for the trace conditioning paradigm but no effect of the lesion of delay conditioning performance. Furthermore, Gould et al. (1999) found an increase of the number of hippocampal neurons in adult rats after trace conditioning, indicating neural plasticity. For those rats that were presented to a delay conditioning paradigm, no changes in hippocampal neuron density could be observed. Thompson (1991) proposes that for simple types of conditioning, hippocampal processing is not necessary, whereas it is essential for complex learning involving declarative memory functions. This interpretation is in good accordance with results from Kishimoto et al. (2001). The authors found an impairment of long interval trace but not delay eyeblink conditioning in mutant mice lacking a specific NMDA receptor. As these receptors are essential for long-term potentiation (LTP) in the hippocampus, the results can be interpreted as reflecting the importance of hippocampal LTP for trace but not delay conditioning. In accordance with this view, others (Clark \& Squire, 1998) have shown that hippocampal functions are not obligatory for simple delay conditioning for which the US occurs when the CS is still present. In contrast, trace conditioning, with a time gap between CS offset and US onset, requires an intact hippocampus. This distinction is further supported by data from studies of brain-injured humans. When delayed conditioning was applied, no differences could be found between patients with hippocampal lesions and healthy controls (e.g. Gabrieli et al., 1995), whereas with trace conditioning an impaired learning of this patient group has been demonstrated (e.g. Daum et al., 1991, for an overview see McGlinchey-Berroth, 2000.) Carrillo et al. (2001) found unaffected discrimination of two tones but impaired reversal learning during a differential conditioning paradigm in patients with a medial temporal lobe lesion. Consistent with Thompson (1991), they argue that the hippocampus is necessary for complex types of learning but not for simple delayed discrimination learning.

In the present MEG study, we applied a differential eyeblink conditioning paradigm. The subjects received two types of $\mathrm{CS}$. The $\mathrm{CS}+$ was paired with the US during the acquisition but not during the extinction, whereas the CS- was not 
paired. The degree of learning was quantified as the difference in $\mathrm{CR}$ rates between the $\mathrm{CS}+$ and the CS- condition. We focused on the intensity as well as on the time course of the cerebellar and hippocampal activation using both a trace and a delay conditioning paradigm.

As evoked neural activity from the cerebellum and the hippocampus has been demonstrated before via MEG (Tesche \& Karhu, 1997; Tesche et al., 1996; Tesche, 1997), we expected to find more activation toward the CS+ than the CS- in the cerebellum for both experimental paradigms. In contrast, the hippocampus should be active only during trace conditioning. Further, in a more explorative approach, we studied the time course of activation to find out whether cerebellar activation precedes or follows hippocampal activation.

\section{EXPERIMENTAL}

\section{Subjects}

Thirty subjects ( 12 males, mean age 25 years, range 20-34 years) participated in the study. Two subjects had to be excluded because of lack of conditioned eyeblink responses. In order to prevent a learning transfer from one experimental paradigm to the other, we used a between-subject design. Fourteen subjects ( 5 males) received a delayed conditioning paradigm, the other 14 subjects (5 males) underwent the trace conditioning paradigm. The two groups did not differ with regard to age or educational level. Informed consent was obtained from all subjects. Each subject received $15 €$ for participation.

\section{Stimulus material and experimental design}

A differential conditioning paradigm was applied. The acquisition phase consisted of $150 \mathrm{CS}+$ trials with a combined CS-US presentation and 150 CS- trials, in which the CS- was presented alone. The subsequent extinction phase consisted of 75 unpaired CS+ and $75 \mathrm{CS}$ - trials. The trial sequence was pseudo-randomized with no more than two equal CS types following each other. The mean inter trial interval (ITI, offset of a trial to onset of the next trial) was $2.5 \mathrm{~s}$, varying between $2 \mathrm{~s}$ and $3 \mathrm{~s}$. This short ITI was chosen to allow a high number of trials per subject, which is necessary to achieve an acceptable signal-to-noise ratio.

Instead of an acoustic stimulation, which is typical for eyeblink conditioning, we used a visual one because aural stimulation would have produced activation in temporal brain regions that would have been difficult to distinguish from hippocampal activity during source localization. In addition, visual stimulation ensured that the subjects would not close their eyes during the experiment. In order to present physically comparable stimuli for the CS+ and CS- we used circles $(10 \mathrm{~cm}$ in diameter) filled with black and white lines that differed only in terms of the orientation of the lines. The stimuli were presented on a 17 -inch computer monitor located $2 \mathrm{~m}$ in front of the subjects outside the shielded MEG room. For one CS stimulus the lines were turned $45^{\circ}$ clockwise and for the other one $45^{\circ}$ counter clockwise. Both stimuli were alternately used as CS+ and CS-, respectively. In the delay conditioning group, the CS was presented for 750 ms and during acquisition immediately followed by the US. For the trace conditioning group, the CS was presented for $100 \mathrm{~ms}$ followed by a 650 ms interval and then, during acquisition, by the US. The US was a 1.48 atmospheres air puff of 50 ms duration presented on one cornea (half of the subject left, half right). The puff was presented via special metal-free goggles in which the air tube was implemented.

The whole head MEG was measured by a 122 channel Neuromag-122 ${ }^{\mathrm{TM}}$ MEG system (Ahonen 
et al., 1993) with a sample rate of $400 \mathrm{~Hz}$ and a band pass filter with a high pass cut off from $.1 \mathrm{~Hz}$ to account for DC like artifacts and a standard low pass cut off at a quarter of the sampling rate. Eyeblinks were measured with two $\mathrm{Ag} / \mathrm{AgCl}$ electrodes attached vertically above and below the stimulated eye, sampled by a Neuroscan Synamps ${ }^{\circledR}$ EEG system (Neuroscan Labs, Sterling, Virginia) and amplified and stored by the MEG system.

Before the experiment, the head of each subject was digitized using 30 landmarks. This information was used to define sensor positions relative to the individual head of the subject, which is essential for group analysis of source activation.

The stimulus presentation and timing was controlled by a PC using STIM software (Neuroscan Labs, Sterling, Virginia), which is especially designed for event-related EEG and MEG studies providing a timing that is adjusted to the refresh cycle of the monitor. The air puffs were produced by a generator developed in our own laboratory and controlled by the STIM software. Due to air compression in the tube, the delivery of the air puff was delayed by $50 \mathrm{~ms}$, which was explored as being constant before the experiment.

\section{Data analysis}

Each trial was manually examined for the existence of a conditioned eyeblink response, which was defined as a reaction occurring between CS and US onset. This is an unusually long-time window to determine CRs. However, alpha or orienting responses as often observed for visual stimuli should contribute to both conditions in the same manner. Therefore, differences between $\mathrm{CS}+$ and CS- should be due to the experimental variation rather than to the stimulation. For each subject, the percentage of eye blinks was calculated for each experimental condition separately. To examine the course of learning for each subject, blocks of 10 trials during acquisition and blocks of 5 trials during extinction were averaged and the percentage of eye blinks per block calculated for each experimental condition separately.

The results were analyzed using a three-way analysis of variance (ANOVA) with the factors 'CS type' (CS+ vs CS-), 'paradigm' (trace vs delay) and 'block' (1 to 15), separately for acquisition and extinction. Additionally, to document the size of extinction effects, a threeway ANOVA was computed with the factors ' $\mathrm{CS}$ type' (CS+ vs CS-), 'paradigm' (trace vs delay) and 'time' (last 50 trials of extinction vs. first 50 trials of extinction),

For all subjects, evoked magnetic activity was averaged for the CS+ and the CS- condition separately. Before averaging, the signal was filtered with a zero phase bandpass filter between 1.5 and $20 \mathrm{~Hz}$ because the evoked magnetic field components were expected within this frequency range. With these evoked magnetic field responses, a source analysis was conducted using Brain Electric Source Analysis (BESA, Scherg, 1990). All MEG analyses were conducted with the BESA 2000 software package (MEGIS Software $\mathrm{GmbH}$, Gräfling, Germany). With respect to the high amount of spread activity in the present paradigm and to the inverse problem of source localization, a unique solution for dipole localization could not be found. Therefore and because our specific regions of interest were defined before the experiment, we used regional source analysis to investigate the activity in the cerebellum and the hippocampus.

With this method, dipoles are defined in specific anatomical regions of interest. To maximize the amount of variance explained, individual source fitting is conducted but restricted to the source orientation. The software calculates the specific signal component of the measured 
data, which is explained by the particular dipole for a predefined time window. Parameters like amplitude (in nAmp) and the latency of the resulting signal can be analyzed. Because the BESA algorithm is sensitive to temporal and spatial interference with other active sources, we defined additional regional sources in regions in which activity can be expected in the present paradigm. These additional sources were located in both eyes to account for eye movements, the bilateral occipital lobes to account for primary and secondary visual processes, the parietal lobe to account for activation from the temporal visual system, and unspecific attentional processes and the vertex to reduce a maximum of unspecific activation.

Figure 1 displays the position of the sources used in the present study. The same source configuration was used for each subject adjusted only with respect to the individual head size and shape. The resulting signals from our four sources of interest (cerebellum and hippocampus, left and right) were analyzed with respect to the maximal peak-to-peak amplitude in the time window 0 to $750 \mathrm{~ms}$ after CS onset. Because the stimulus presentation side was counterbalanced between subjects, the resulting activation from the two sources per region was averaged, resulting in one activation value per region. Latencies were defined as time between CS onset and the peak of the maximal amplitude. The differences between groups were statistically confirmed using a threeway mixed model ANOVA with the within subject factors 'CS-type' (CS+ vs. CS-) and 'region' (cerebellum vs. hippocampus) and the between subject factor 'paradigm' (delay vs. trace). Analyses of amplitudes and latencies were conducted for acquisition and extinction separately.
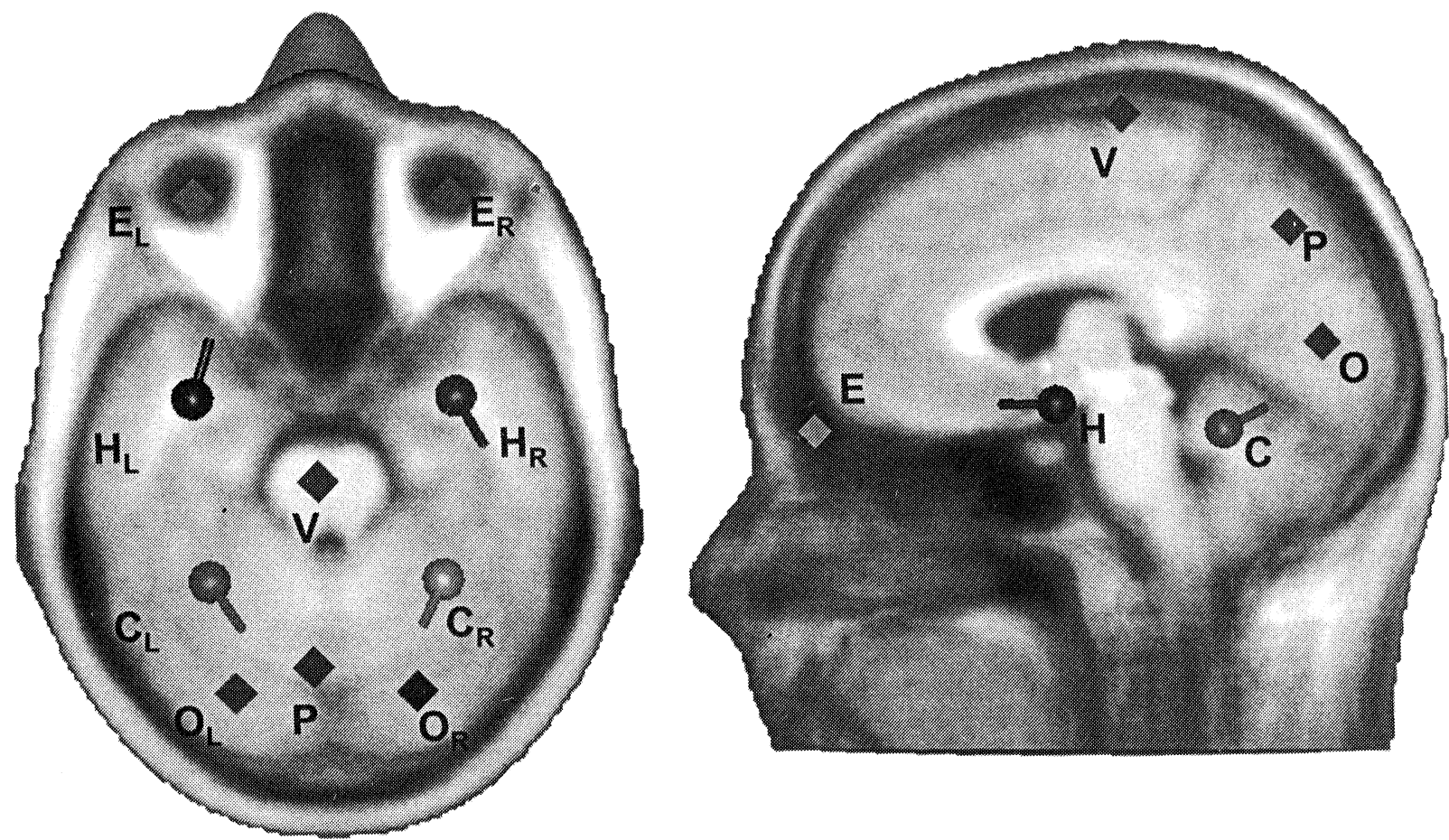

Fig. 1: Configuration of the dipoles of interest and the additional regional sources superimposed on a standardized brain image. Abbreviations are $\mathrm{C}$ for Cerebellum, $\mathrm{H}$ for Hippocampus, $\mathrm{E}$ for Eyes, $\mathrm{O}$ for occipital site, $\mathrm{V}$ for vertex and $\mathrm{P}$ for parietal site. Additional characters $\mathrm{L}$ and $\mathrm{R}$ indicate left and right sources. 


\section{RESULTS}

\section{Conditioned eyeblink responses}

Subjects showed significantly more eyeblinks to $\mathrm{CS}+$ than to $\mathrm{CS}-$ during acquisition $(\mathrm{F}(1 / 26)=$ 2.694, $\mathrm{p}<.001)$. The difference between $\mathrm{CS}+$ and CS- increased over time, indicated by a significant CS-type by block interaction $(\mathrm{F}(14 / 364)=8.188$, $\mathrm{p}<.001, \varepsilon=.396$, Fig. 2), but no difference was found between trace and delay conditioning. Thus, comparable conditioning effects were found for both groups.

The extinction was very fast as reflected by a significant time main effect $(F(1 / 26)=175.353$, $\mathrm{p}<.001$ ), as well as by a CS-type by time interaction $(F(1 / 26)=3.943, p<.001)$ for ANOVA comparing the last 50 acquisition trials with the first 50 extinction trials (Fig. 3.) While there was a highly significant $\mathrm{CS}+/ \mathrm{CS}$ - difference during the end of acquisition $(p<.001$ for the post-hoc $t$ -
Test), the difference between CS+ and CS- nearly disappeared for the extinction $(p<.06$, onesided.).

During extinction, there were no differences between the CS+ and CS- condition with respect to the percentage of eyeblinks. However, the interaction between CS-type and paradigm showed a trend towards significance $(\mathrm{F}(1 / 26)=3.281$, $\mathrm{p}<.09)$. The post-hoc t-test revealed significantly more eyeblinks to the CS+ than the CS- only for the trace but not for the delay group (Fig. 4).

\section{Magnetic brain activity}

The source model used for the analysis of brain activation explained between $63.9 \%$ and $78.2 \%$ of the variance of the MEG signal. The ANOVA of the source activation amplitude during acquisition revealed a significant three-way interaction $(\mathrm{F}(1 / 26)=4.242, \mathrm{p}=.05)$. As displayed in Fig. 5, stronger source activation during CS+

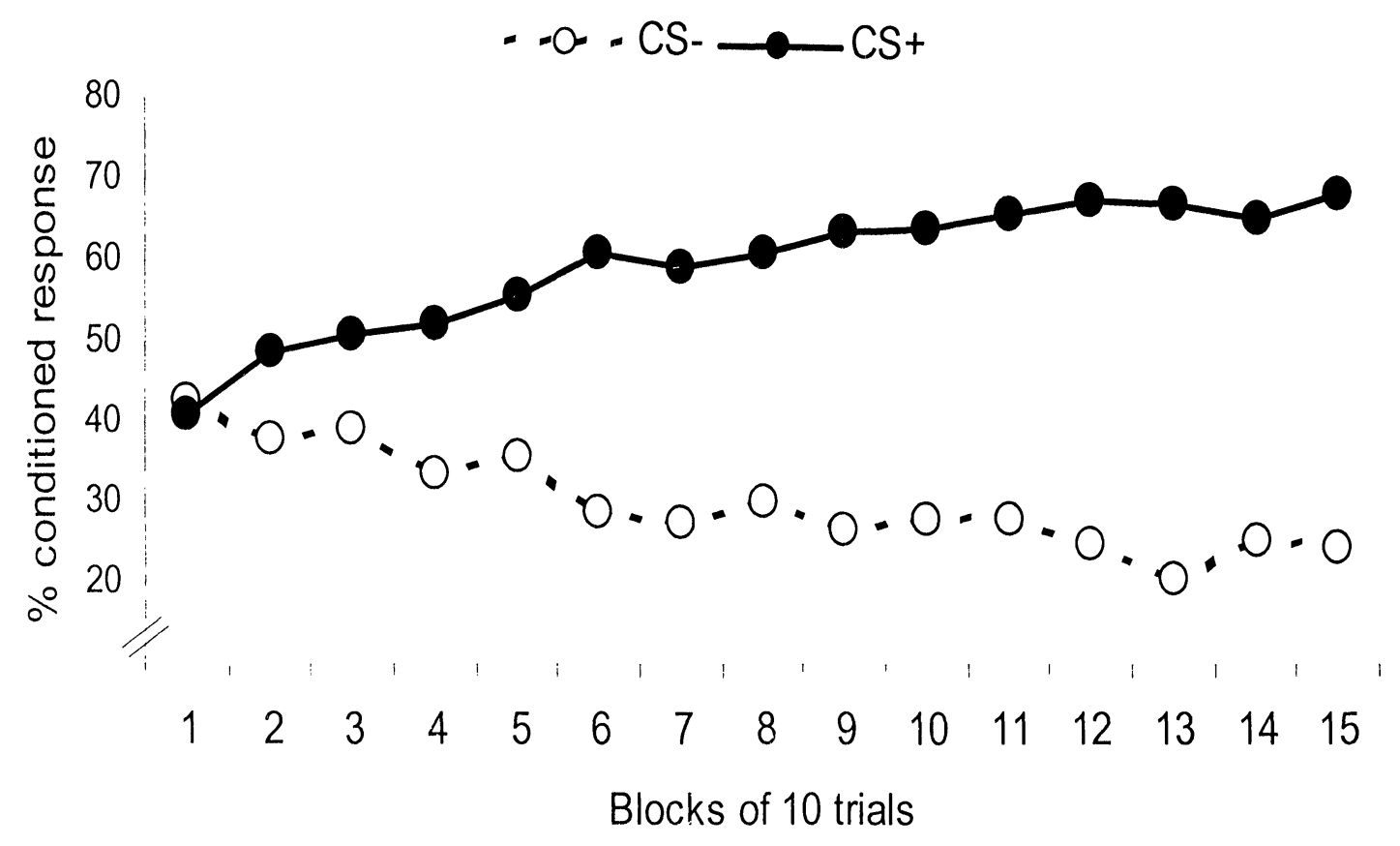

Fig. 2: Mean percentage of conditioned eyeblink response to the CS- (dotted line) and the CS+ (solid line) during acquisition for all subjects, averaged over 10 trials per block. 


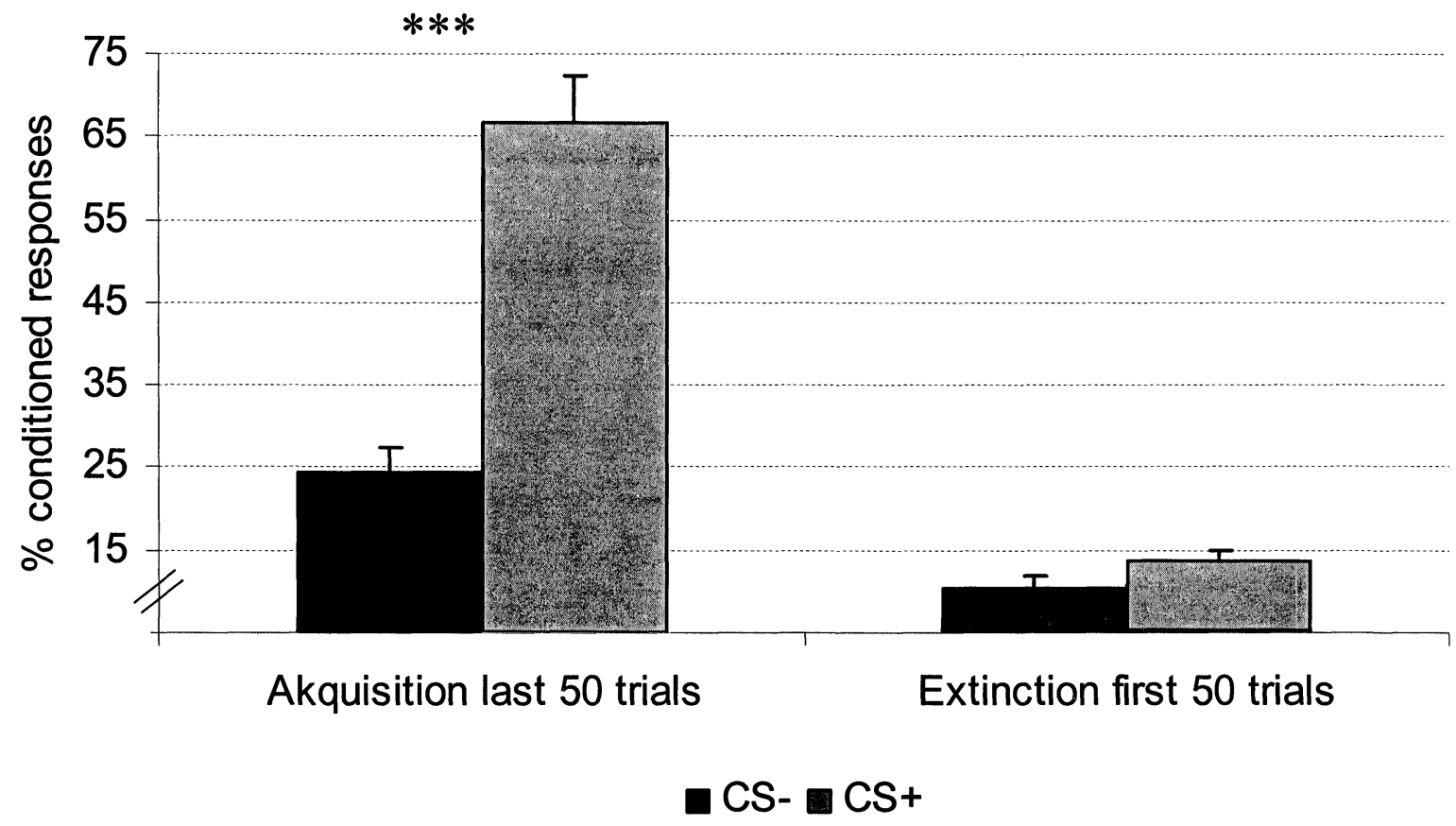

Fig. 3: Mean percentage (and S.E.M.) of conditioned eyeblink response from all subjects to the CS- (black bars) and the CS+ (gray bars) during the last 50 trials of acquisition on the left side and the first 50 trials of extinction on the right side. (comparison between CS- and CS+: $* * *=p<.001$, two-sided).

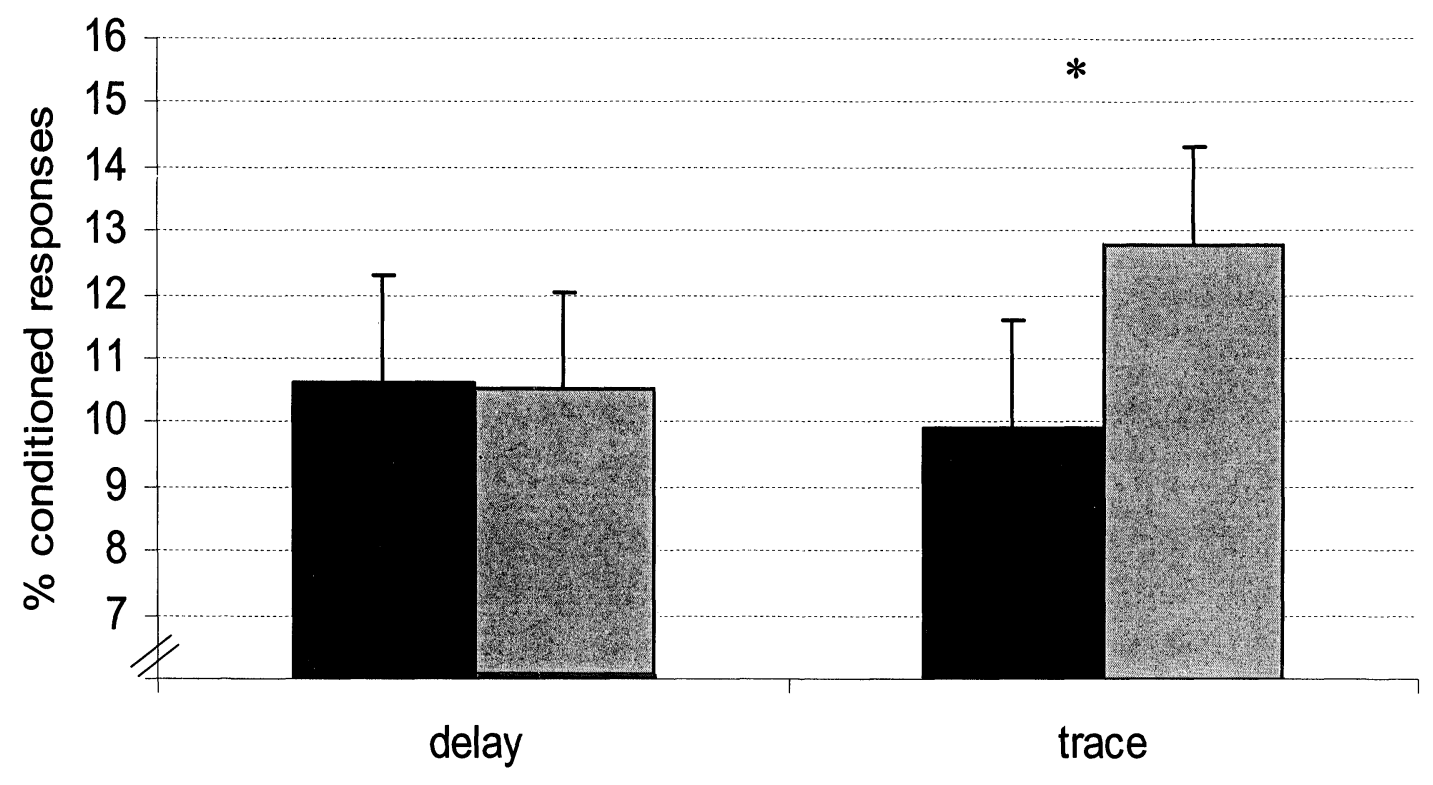

- CS- CS+

Fig. 4: Mean percentage (and S.E.M.) of conditioned eyeblink response to the CS- (dark bars) and the CS+ (light bars) during extinction. Mean responses of the delay group are displayed on the left and of the trace group on the right side. (comparison between $\mathrm{CS}-$ and $\mathrm{CS}+:^{*}=\mathrm{p}<.05$, two-sided). 
than CS- presentation was found in the delay conditioning group only in the cerebellum. In contrast, for the trace conditioning group a stronger activation to the CS+ than to the CSoccurred only in the hippocampus. No significant differences were found for the extinction.

Analyses of the latency of cerebellar and hippocampal activation during acquisition revealed a significant main effect for the region $(F(1 / 26)=$ $6.69, \mathrm{p}<.02)$. There was an earlier activation of the cerebellum (mean peak latency $=179.78 \mathrm{~ms}$ ) compared with the hippocampal sources (mean peak latency $=221.32 \mathrm{~ms}$ ). No significant main effect of CS type or experimental paradigm and no significant interaction were found. A similar regional main effect was found for the extinction
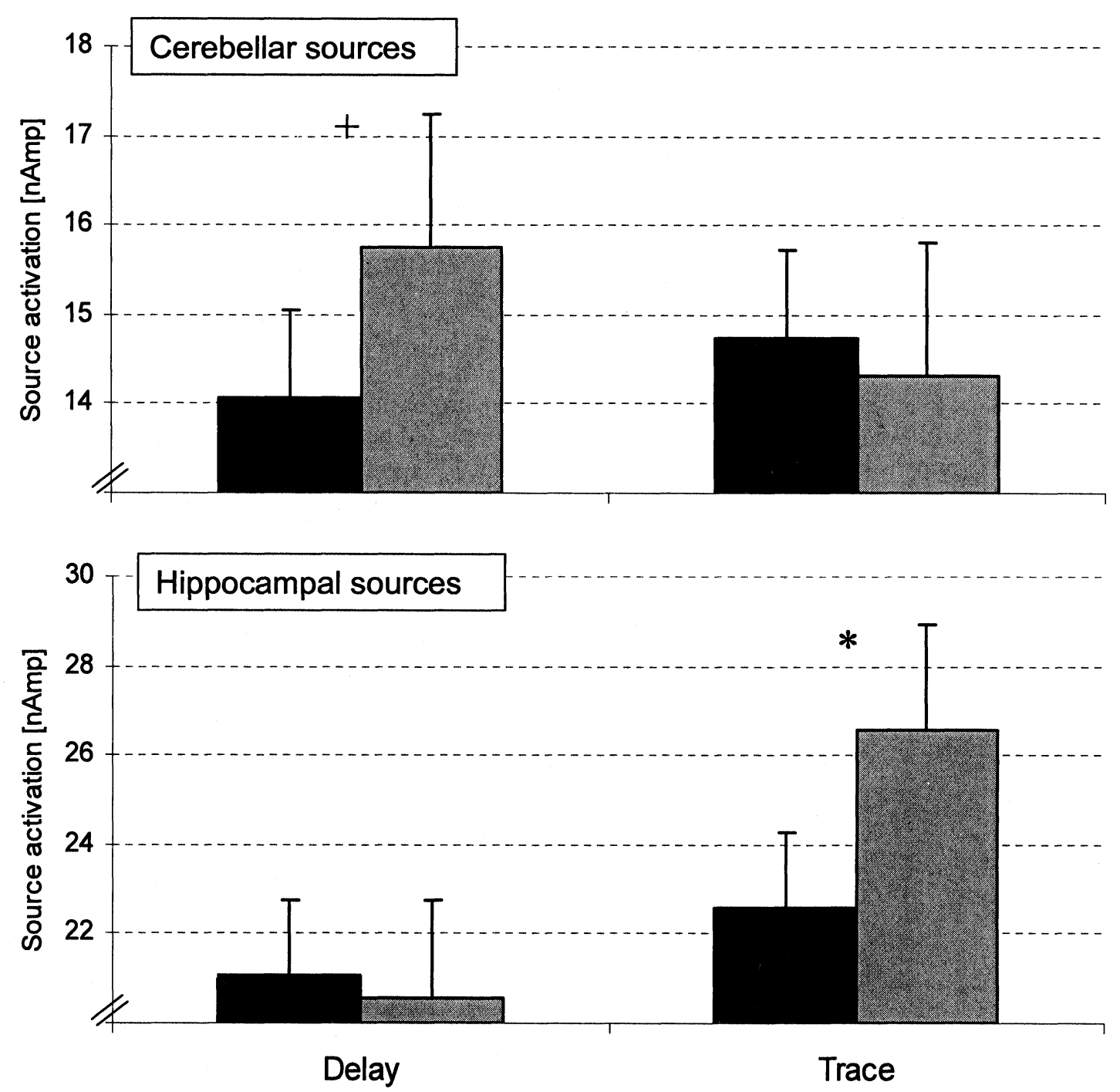

CS- $\square$ CS+

Fig. 5: Mean source activation (and S.E.M.) at CS- (dark bars) and CS+ presentation (light bars) during acquisition for the cerebellar (upper panel) and the hippocampal sources (lower panel). Mean activations of the delay group displayed on the left and of the trace group on the right side (comparison between CS- and CS+: ${ }^{*}=p<.05 ;+=p<.1$, two-sided). 
$(\mathrm{F}(1 / 26)=7.44, \mathrm{p}<.02)$, again with a faster activation of the cerebellum (mean peak latency $=$ $190.92 \mathrm{~ms}$ ) than the hippocampus (mean peak latency $=246.0 \mathrm{~ms}$ ).

\section{DISCUSSION}

The results of the present study demonstrate that classical eyeblink conditioning can be investigated using MEG methodology. In both experimental conditions, comparable differential eyeblink conditioning occurred. The relatively flat learning curve (Fig. 2), could be a result of the unusual short ITI used in the present study. There is some evidence that in animal studies an ITI from at least $10 \mathrm{~s}$ is necessary to establish stable conditioning (Nordholm, et al., 1991). However, successful eyeblink conditioning was demonstrated with shorter ITIs in humans. Carrillo et al. (1997) reported no difference in the conditioned response rate between ITIs of 5,10 , or 30 seconds. Furthermore, our conditioned response rates observed for the last 50 trials during acquisition, as displayed in Fig. 3, are comparable to those of other studies with normal participants. Therefore, we conclude that we were able to initiate differential eyeblink conditioning in the present study.

In contrast to the acquisition, delay and trace conditioning differed in terms of resistance to extinction. While the differentiation between $\mathrm{CS}+$ and CS-completely disappeared in the delay group, there were slightly more CRs to the CS+ than the CS- in the trace group. This effect cannot be attributed to a sensitization to CS- during delay conditioning, since the number of CRs to CS- did not increase and the number of CRs to CS+ decreased. Even when we take into account that the absolute number of CRs to $\mathrm{CS}+$ during trace conditioning was rather small, our results confirm that the CS-US association learned in this condition was more stable than during delay conditioning. This result might be due to hippocampal processing, possibly related to awareness of the CS-US association (Clark, \& Squire, 1998; Manns et al, 2000), leading to a longer lasting memory for this association. This hippocampal involvement during trace conditioning was confirmed by analysis of the magnetic field activity. Only for trace conditioning more activation to the CS+ than the CS- was found in the hippocampus. This result is in good accordance with our hypothesis. Also in line with our hypothesis was the stronger cerebellar activation to the CS+ than the CS- in the delay conditioning group.

In contrast to our expectations was the lack of differentiation between CS+ and CS- for cerebellar activation during trace conditioning. All current models of eyeblink conditioning emphasize the central role of the cerebellum for this phenomenon. However, it could be argued that some cerebellar functions were taken over by the hippocampus during trace conditioning. Kishimoto and colleagues (2001) observed an impaired delay conditioning but an unaffected trace eyeblink conditioning in PCLbeta4 mutant mice. Such mice showed a reduced long-term depression (LTD) in the rostral cerebellum, which might be essential for delay but not for trace conditioning. Although we have no evidence for the conclusion that our source activation reflects LTD, the results demonstrate that delay and trace conditioning might differ in terms of cerebellar involvement.

As a shortcoming of our study, we have to mention that we used only one source per cerebellar hemisphere. Therefore, we cannot be sure that all cerebellar processes during our experimental procedures were registered to the same degree. Furthermore, increases in activity of visual systems could have been wrongly explained by the cerebellar sources during trace conditioning. Although we had applied occipital as well as 
parietal sources to explain most of this sensory activity, we cannot completely exclude that visual activity was absorbed by the cerebellar sources. While differences between CS+ and CS- during delay conditioning cannot be attributed to visual processing of physically identical stimuli and should reflect cerebellar processing, the lack of difference between the CS conditions during trace conditioning could be based on a wrong localization of visual activity, which in fact should not differ between CS+ and CS-.

The analysis of the latency of the source activation did not reveal any differences between the experimental conditions. Neither the CS type nor the paradigm influenced the onset of activation. However, we found a significant main effect of structure with an earlier activation of cerebellar than hippocampal sources for all conditions and for acquisition as well as extinction. These findings make a lot of sense for delay conditioning, as it could be argued that the cerebellum can independently controls the conditioned response. Hippocampal activation could reflect attentional processing of the stimuli without a crucial influence on the CR. In contrast, during trace conditioning, hippocampal processing should influence the $C R$ initiation through the cerebellum. Therefore, cerebellar activation should follow the hippocampal activation rather than precede it. However, since we found no specific cerebellar activation during trace conditioning, it might reflect more learningindependent processes or, as mentioned before, activation from other regions.

A method with a better spatial resolution like fMRI could help to find differences as well as congruencies in cerebellar activation between delay and trace conditioning. Furthermore, it would be very interesting to use spatial information revealed by fMRI to improve the source configuration for MEG. As mentioned before, it could also be useful to apply different cerebellar sources for delay and trace conditioning. Thus, the combination of fMRI and MEG methodology promises a deeper understanding of temporal and spatial differences in brain activation during human trace and delay eyeblink conditioning.

\section{ACKNOWLEDGMENT}

This work was supported by a research grant from the Deutsche Forschungsgemeinschaft to PK (Ki 576/4-1).

\section{REFERENCES}

Ahonen, AI. Hämäläinen, MS, Knuutila, JET, Kajola, MJ, Laine, PP, Lounasmaa, OV, et al. 1993. 122channel SQUID Instrument for Investigating the Magnetic Signals from the Human Brain. Physica Scripta T49 : 198-205.

Berger TW, Rinaldi PC, Weisz DJ, Thompson RF. 1983. Single-unit analysis of different hippocampal cell types during classical conditioning of rabbit nictitating membrane response. J Neurophysiol 50: 1197-1219.

Blaxton TA, Zeffiro TA, Gabrieli JD, Bookheimer SY, Carrillo MC, Theodore WH, et al. 1996. Functional mapping of human learning: a positron emission tomography activation study of eyeblink conditioning. J Neurosci 16: 4032-4040.

Bracha V, Zhao L, Wunderlich DA, Morrissy SJ, Bloedel JR. 1997. Patients with cerebellar lessions cannot acquire but are able to retain conditioned eyeblink reflexes. Brain 120: 14011413.

Carrillo MC, Gabrieli JDE, Hopkins RO, McGlinchey-Berroth R, Fortier CB, et al. 2001. Spared discrimination and impaired reversal eyeblink conditioning in patients with temporal lobe amnesia. Behav Neurosci 115: 1171-1179.

Carrillo MC, Thompson LT, Gabrieli JDE, Disterhoft JF. 1997. Variation of the intertrial interval in human classical conditioning. Psychobiology 25: 152-157.

Clark RE, Squire LR. 1998. Classical conditioning and brain systems: The role of awareness. Science 280: 77-81. 
Daum I, Channon S, Polkey CE, Gray JA. 1991. Classical conditioning after temporal lobe lesions in man: impairment in conditional discrimination. Behav Neurosci 105: 396-408.

Daum I, Schugens MM, Ackermann H, Lutzenberger W, Dichgans J, Birbaumer N. 1993. Classical conditioning after cerebellar lesions in humans. Behav Neurosci 107: 748-756.

Gabrieli JD, McGlinchey-Berroth R, Carrillo MC, Gluck MA, Cermak LS, Disterhoft JF. 1995. Intact delay-eyeblink classical conditioning in amnesia. Behav Neurosci 109: 819-727.

Gerwig M, Dimitrova A, Kolb FP, Maschke M, Brol B, Kunnel A, et al. 2003. Comparison of eyeblink conditioning in patients with superior and posterior inferior cerebellar lesions. Brain 126: 7194.

Gould E, Beylin A, Tanapat P, Reeves A, Shors TJ. 1999. Learning enhances adult neurogenesis in the hippocampal formation. Nat Neurosci 2: 260-265.

Kishimoto Y, Hirono M, Sugiyama T, Kawahara S, Nakao K, Kishio M, et al. 2001. Impaired delay but normal trace eyeblink conditioning in PLCbeta4 mutant mice. NeuroReport 12: 29192922.

Kishimoto Y, Kawahara S, Mori H, Mishina M, Kirino Y. 2001. Long-trace interval eyeblink conditioning is impaired in mutant mice lacking the NMDA receptor subunit epsilon1. Eur $\mathbf{J}$ Neurosci 13: 1221-1227

Logan CG, Grafton ST. 1995. Functional anatomy of human eyeblink conditioning determined with regional cerebral glucose metabolism and positron-emission tomography. Proc Natl Acad Sci USA 92: 7500-7504.

Manns JR, Clark RE, Squire LR. 2000. Parallel acquisition of awareness and trace eyeblink classical conditioning. Learn Mem 7: 267-272.

McGlinchey-Berroth R. 2000. Eyeblink classical conditioning in amnesia. In: Woodruff-Pak D, Steinmetz JE, eds, Eyeblink classical conditioning: Volume I: Applications in humans, Boston: Kluwer Academic Press, 205-228.
Nordholm AF, Lavond DG, Thompson RF. 1991. Are eyeblink responses to tone in the decerebrate, decerebellate rabbit conditioned responses? Behav Brain Res 44: 27-34.

Ramnani N, Toni I, Josephs O, Ashburner J, Passingham RE. 2000. Learning- and expectation-related changes in the human brain during motor learning. J Neurophysiol 84: 3026-3035.

Scherg M. Fundamentals of dipole source potential analysis. 1990. In: Grandori F, Hoke M, Romani GL, eds. Auditory Evoked Magnetic Fields and Potentials. Advanced of Audiology vol 6. Basel, Switzerland: Karger 40-69.

Schreurs BG, McIntosh AR, Bahro M, Herscovitch P, Sunderland T, Molchan SE. 1997. Lateralization and behavioral correlation of changes in regional cerebral blood flow with classical conditioning of the human eyeblink response. J Neurophysiol 77: 2153-2163.

Steinmetz JE. 2000. Brain substrates of classical eyeblink conditioning: a highly localized but also distributed system. Behav Brain Res 110: 13-24.

Tesche CD. 1997. Non-invasive detection of ongoing neuronal population activity in normal human hippocampus. Brain Res 749: 53-60.

Tesche CD, Karhu J. 1997. Somatosensory evoked magnetic fields arising from sources in the human cerebellum. Brain Res 744: 23-31.

Tesche CD, Karhu J, Tissari SO. 1996. Non-invasive detection of neuronal population activity in human hippocampus. Cogn Brain Res 4: 39-47.

Thompson RF. 1991. Are memory traces localized or distributed? Neuropsychologia 29: 571-582.

Thompson RF, Krupa DJ. 1994. Organization of memory traces in the mammalian brain. Annu Rev Neurosci 17: 519-549.

Weiss C, Bouwmeester H, Power JM, Disterhoft JF. 1999. Hippocampal lesions prevent trace eyeblink conditioning in the freely moving rat. Behav Brain Res 99: 123-132

Woodruff-Pak DS, Steinmetz JE, eds. 2000. Eyeblink Classical Conditioning: Volume II: Animal Models. Boston, Massachusetts, USA: Kluwer Academic. 

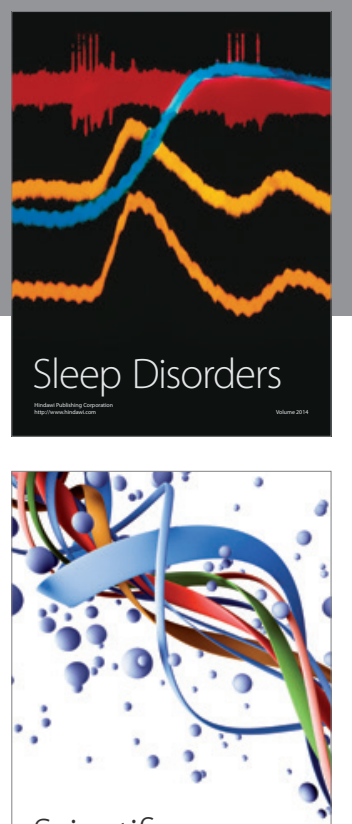

Scientifica
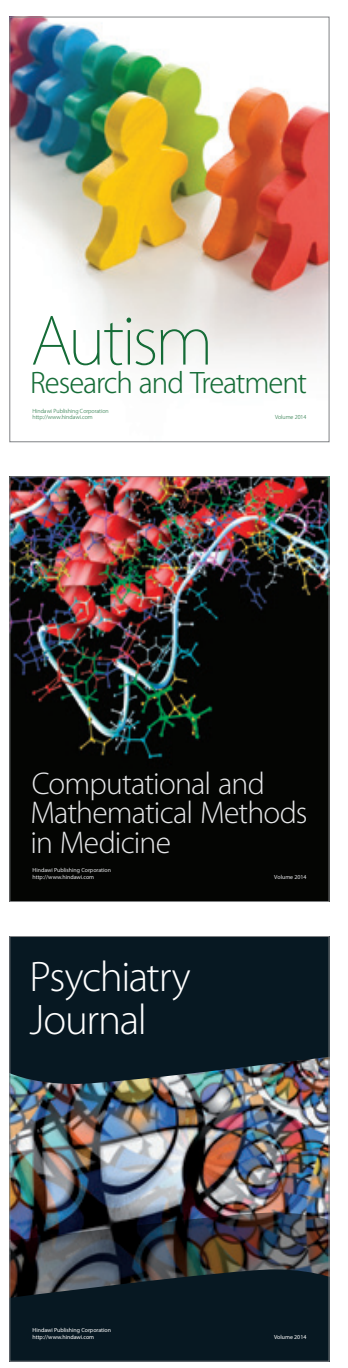
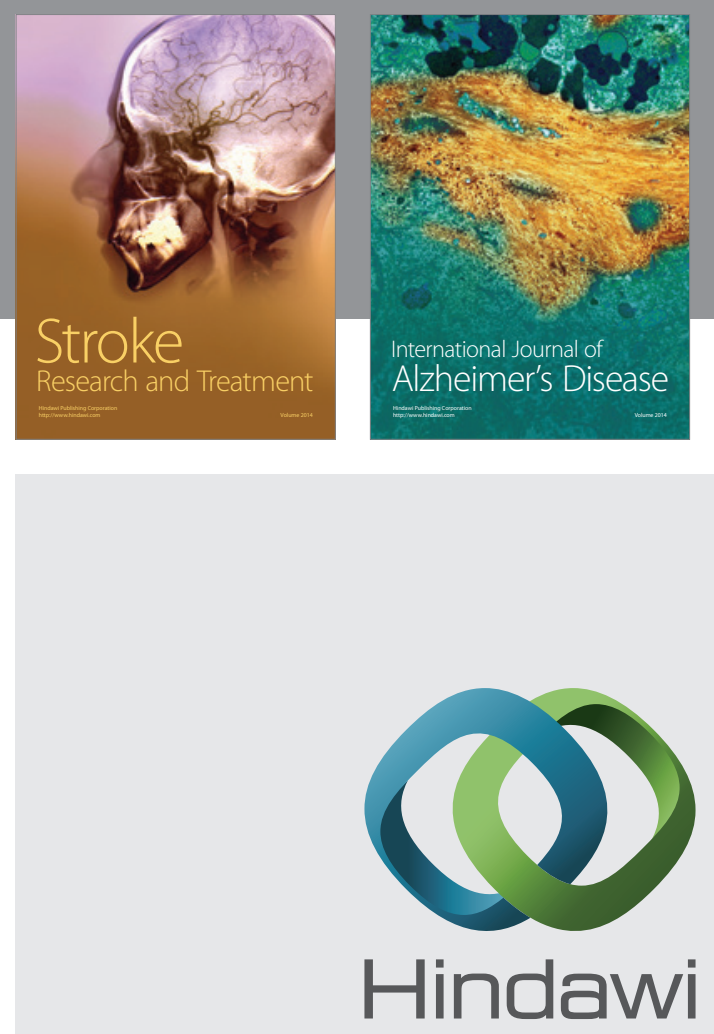

Submit your manuscripts at

http://www.hindawi.com
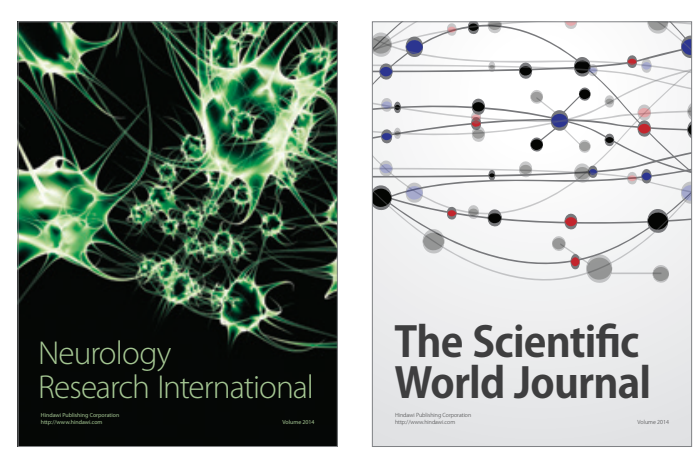

The Scientific World Journal

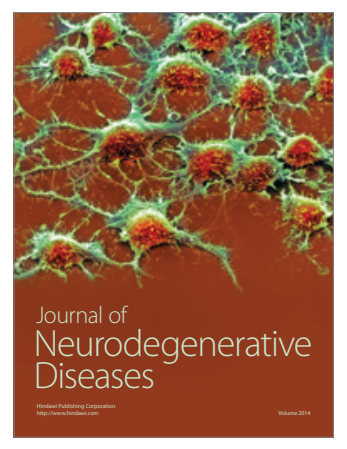

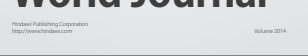

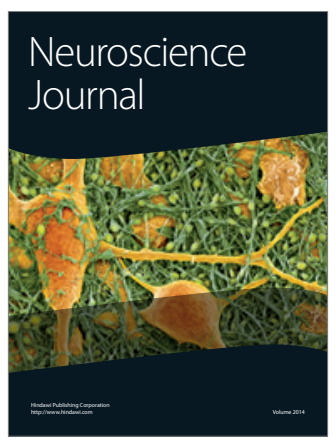

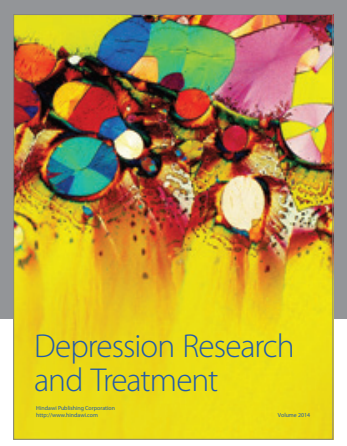
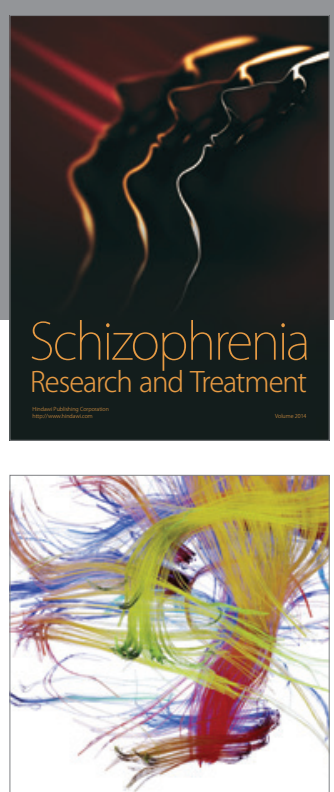

Brain Science

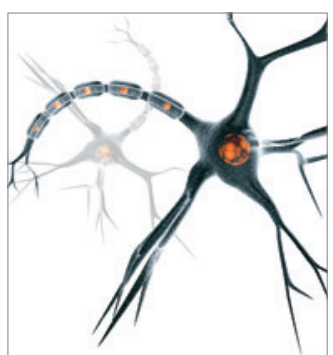

Neural Plasticity
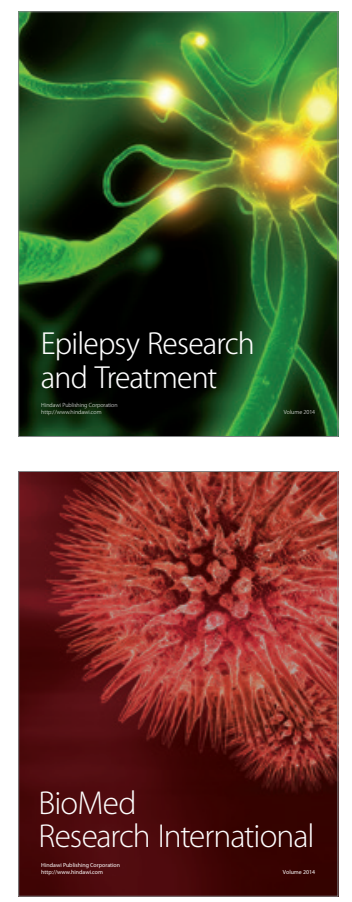

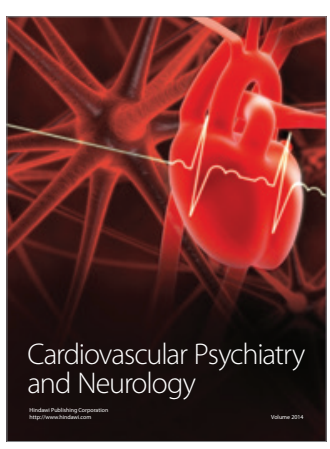

Parkinson's

Disease
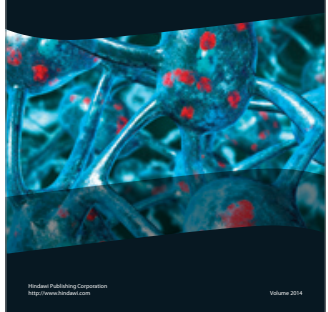